THE EFFECT OF Li, He AND Ca ON GRAIN BOUNDARY COHESIVE STRENGTH IN Ni

\author{
Richard W. Smith ${ }^{1}$, W. T. Geng ${ }^{2}$, Clint B. Geller ${ }^{1}$, R. Wu ${ }^{3}$, A. J. Freeman ${ }^{2}$
}

1) Bettis Atomic Power Laboratory

Bechtel Bettis Inc.

West Mifflin, PA 15122

2) Northwestern University

Evanstron, IL 60208

3) California State University

Northridge, CA 91330

This report was prepared as an account of work sponsored by the United States Government. Neither the United States, nor the United States Department of Energy, nor any of their employees, nor any of their contractors, subcontractors, or their employees, makes any warranty, express or implied, or assumes any legal liability or responsibility for the accuracy, completeness or usefulness of any information, apparatus, product or process disclosed, or represents that its use would not infringe privately owned rights. 


\title{
THE EFFECT OF Li, He AND Ca ON GRAIN BOUNDARY COHESIVE STRENGTH IN Ni
}

\author{
Richard W. Smith ${ }^{1}$, W. T. Geng ${ }^{2}$, Clint B. Geller ${ }^{1}$, R. Wu ${ }^{3}$, and Arthur J. Freeman ${ }^{2}$
}

1) Computational Materials Analysis Group, Bettis Atomic Power Laboratory, West Mifflin, PA 15122.

2) Department of Physics and Astronomy, Northwestern University, Evanston, IL 60208.

${ }^{3)}$ Department of Physics and Astronomy, California State University, Northridge, CA 91330.

Keywords: ab initio calculation; grain boundaries; embrittlement; nickel alloys; FLAPW

\section{Introduction}

Boron is added to nickel-base superalloys such as Alloy $X-750$ in order to enhance high temperature strength and ductility so that the alloy may be more easily hot worked [1]. Boron additions also have been shown to ameliorate intergranular hydrogen embrittlement in nickel [2], and to improve the high temperature resistance of Alloy X-750 to aqueous stress corrosion cracking (SCC) in the absence of irradiation [3]. Recent quantum mechanical calculations demonstrate that boron strengthens grain boundaries in pure nickel [4], and may contribute to the observed benefits of boron on workability and fracture resistance of nickel alloys. Unfortunately, when X-750 is used under conditions of high neutron flux, an $(n, \alpha)$ reaction $\left(B^{10}+n^{1} \rightarrow \mathrm{Li}^{7}+\mathrm{He}^{4}\right)$ transmutes some of the $\mathrm{B}^{10}$ at the grain boundaries to lithium and helium. (The isotopic abundance of $\mathrm{B}^{10}$ is roughly $20 \%$ in natural boron.) Alloy X-750 exhibits greater susceptibility to intergranular stress corrosion cracking (IGSCC) when irradiated [5], and it has been proposed that the presence of grain boundary helium and/or lithium is responsible. Arguments have been advanced that helium embrittlement of the grain boundaries is primarily responsible for the greater observed susceptibility to IGSCC in irradiated X-750 [1]. Alternatively, it has been proposed that lithium promotes IGSCC either by entering the water at the crack tip and lowering the local $\mathrm{pH}$, or by inducing a restructuring of the grain boundary itself [1]. Direct embrittlement of grain boundaries by lithium also has been investigated by ion bombardment in Nimonic PE16, illustrating that under certain conditions lithium can produce degrees of embrittlement in nickel comparable to that produced by helium [6]. It is important to understand the relative roles of these species in grain boundary embrittlement in nickel alloys so that better predictive abilities and mitigation strategies can be developed. Toward that end, quantum mechanical calculations have been performed to investigate the influence of isolated lithium and helium atoms on the cohesive strength of an ideal grain boundary in pure nickel.

In a related area, calcium is added in the form of lime $(\mathrm{CaO})$ to the molten nickel base alloys in order to getter sulfur and thereby reduce intergranular embrittlement. $\mathrm{CaO}$ reacts with elemental $\mathrm{S}$ to form stable precipitates of $\mathrm{CaS}$ that are removed from the material in slag. Minor amounts of $\mathrm{CaO}$ and $\mathrm{CaS}$ that may remain in the alloys are thought to exist in the form of stable precipitates that are chemically inert. Nevertheless, in the event that an as yet unrealized environmental condition may transport some of the $\mathrm{Ca}$ to the grain boundary in an elemental state, it is important to assess the worst case effect of the element on grain boundary cohesion. This assessment is made with the same quantum mechanical methodology. 


\section{Computational Method}

All calculations discussed in this report were performed using the full potential linear augmented plane wave (FLAPW) electronic band structure code [7] that can be used to model systems with periodic translational symmetry in all three, or only two of their three dimensions. (The surfaces and interfaces studied in the present work were modeled as 2D-periodic structures.) FLAPW solves the effective single-particle semi-relativistic wave equations for bulk crystals, surfaces or interfaces. From these wave functions and their respective energy eigenvalues, binding energies, cohesive energies, elastic properties, electronic charge distributions and other properties of interest can be determined. The calculations reported herein were performed in the generalized gradient approximation (GGA) level of theory [8]. The GGA tends to be slightly more accurate than its predecessor, the local density approximation (LDA), for systems, such as those containing free surfaces, in which the local charge density exhibits large spatial variations.

Ferromagnetic nickel, with a spin-polarized band structure, rather than paramagnetic nickel was modeled for all impurities except for calcium, because the ferromagnetic case is easier to converge computationally. The calcium impurity was modeled both ferromagnetically and paramagnetically, to provide a measure of how much the assumed magnetic state would affect the energy parameters of interest. It was found that the calcium binding energies in the grain boundary and on the free surface each differed by less than $7 \%$ between the ferromagnetic and paramagnetic cases, resulting in a strengthening energy difference between these two magnetic cases of less than $1 \%$. This result was expected since the magnetic energy density in nickel is low compared with that of other ferromagnetic elements like iron and cobalt.

Predictions of impurity effects on grain boundary cohesion are based on the thermodynamic theory of Rice and Wang [9]. This theory relates the change in the overall cohesive strength of a grain boundary (GB) to the binding energies of the GB and the free surfaces (FS) that would be formed by cleaving the GB, with and without the impurity (Fig. 1). The key quantity to be calculated in this theory is the Griffith Work change, also called the "Strengthening Energy." The Griffith Work change is given by
Eq. 1
$\Delta \mathrm{E}_{\mathrm{GW}}=\Delta \mathrm{E}_{\mathrm{b}}-\Delta \mathrm{E}_{\mathrm{s}}$

where $\Delta \mathrm{E}_{\mathrm{b}}$ and $\Delta \mathrm{E}_{\mathrm{s}}$ are the impurity atom binding energies in the GB and in the FS, respectively:

Eq. 2

$$
\begin{aligned}
\Delta E_{b} & =E(G B)-E(I / G B)+E(I) \\
\Delta E_{s} & =1 / 2[E(F S)-E(l / F S)]+E(I)
\end{aligned}
$$

Eq. 3

In equations 2 and $3, E(I / G B)$ and $E(G B)$ are the total energies of the $G B$ with and without the impurity, respectively; $E(I / F S)$ and $E(F S)$ are the total energies of the $F S$ with and without the impurity, respectively; and $E(I)$ is the total energy of a monatomic line of impurity atoms. The factor of one half appears in Equation 3 because the FS unit cell chosen for the calculation includes two impurity atoms.

Griffith Work values are determined as total electronic energy differences per impurity atom between slabs of material respectively representing two-dimensional arrays of grain boundaries and free surfaces with and without impurities. In the current investigation, a nickel $\Sigma 5(210)$ GB was modeled using a two-dimensional unit cell previously described by Geng et al. [4]. Atomic positions within the unit cell are optimized via a self-consistent force minimization procedure. The geometry optimization procedure for these calculations positions the outer layers of atoms in the unit cells such that the net force on the unit cell is zero, in order to simulate an infinite crystal or surface. Both in-plane and outof-plane distortions were permitted. The convergence of the geometry with unit cell size has been studied sufficiently to be confident that the effects of geometric relaxation, as modeled, are relatively close to their limiting, infinite unit cell values. 
Calcium is a large, bivalent ion unlike any impurity previously modeled on a nickel grain boundary, and thus, the current study of $\mathrm{Ca}$ was informative. The reduction in grain boundary binding energy as a result of the presence of a calcium impurity as an interstitial atom within the grain boundary was so large, it was concluded that atomic calcium would be unlikely ever to occupy such a position in a real material. The large reduction in GB binding energy results because calcium has an atomic radius (1.97 Angstroms) 58\% larger than that of nickel (1.24 Angstroms). Hence, it was considered more likely that such an oversized atom would occupy a substitutional position at or near, rather than in a nickel grain boundary. This case also was investigated.

\section{Results and Discussion}

The cumulative strengthening energy results of the currently reported study and that of Geng et al. [4] are summarized in Table I. The predictions for hydrogen, phosphorus and boron, which are respectively, two known embrittlers and a known strengthener, provide confidence in the current computational method. The results for lithium are mirrored qualitatively in the earlier results for hydrogen. For both, the net effect of these species is not to increase the binding energy of the grain boundary relative to the free surface, but to disrupt the metallic bonds that would otherwise exist between adjacent nickel atoms across a clean GB. Helium binds neither to the grain boundary nor to the free surface. Hence, it is clear that other than mechanical distortion effects, which always weaken grain boundaries, a helium impurity can act only to interfere with chemical bonds that would exist in its absence. The large strengthening energy $(2.5 \mathrm{eV})$ associated with helium is mainly due to the screening of the nickel atom wave functions sited on opposite sides of the helium charge cloud and the resulting disruption or weakening of the nickel-nickel bonds $(\sim 2.0 \mathrm{eV})$.

The calculations illustrate that both helium and lithium, as isolated atoms at nickel grain boundaries, are capable of causing significant embrittlement. The most immediate implication of this result is that neither species can be dismissed as harmless based on its intrinsic atomic interaction with the host material. Another important point is that either species can degrade the cohesion of the boundary simply by being present in sufficient quantity. Embrittlement does not require elaborate mechanisms involving collective effects such as helium agglomeration into bubbles, liquid metal embrittlement due to lithium, or lithium effects on water chemistry. The current study does not show that such mechanisms do not operate or cannot exacerbate the problem, but merely that they may not be necessary to explain observed behavior. As a result, it is clear that full mitigation of the embrittlement problem would require not only impeding the operation of the collective processes, but also the elimination of both helium and lithium in any form from the boundary. Because the present calculations only describe the effects of impurities upon their arrival at the boundary, a complete description of the embrittlement would require, at a bare minimum, additional calculations for the transport and trapping of both species in and around various types of boundaries.

Computational results for calcium show that if free atomic calcium were present on a nickel GB, it could be expected to exert a strong embrittling effect. However, the solubility of calcium in nickel is extremely low and calcium is added to the alloy in the form of $\mathrm{CaO}$, which is a highly stable compound with a melting temperature far above that of any processing temperature seen by the alloy. (CaO has a melting temperature of $2613^{\circ} \mathrm{C}$, and the CaS that forms from it melts at $2525^{\circ} \mathrm{C}$.) Consequently, the use of lime getters alone is not expected to introduce free calcium either to the grain boundaries or to the bulk. 


\section{Summary}

Assuming a linearity between embrittlement potential and the calculated energy differences in Table I, the current computational results indicate that atom for atom, lithium is approximately four times and helium is about eight time more embrittling in a nickel GB than hydrogen. The results also imply that both species must be considered when attempting to determine conditions under which embrittlement would be expected and that models describing the transport of both species in and around the grain boundaries are needed. Results also show that if appreciable levels of free calcium were to accumulate at grain boundaries in a nickel alloy, there would likewise be a potential for enhanced brittle fracture susceptibility.

\section{References}

1. E. E. Brown, D. R. Muzyka, Superalloys II, (John Wiley \& Sons, 1987), p. 165.

2. Y. Ogino, T. Yamasaki, Scripta Met. 15 (1981) 821.

3. C. Benhamou, J. Daret, J. C. Van Duysen, Contribution des Expertises sur Materiaux a la Resolution des Problemes Rencontres dans les REP, Vol 1, (SFEN, Paris, 1990), p.30.

4. W. T. Geng, A. J. Freeman, R. Wu, C. B. Geller, J. E. Raynolds, Phys. Rev. B60 (1999) 7149.

5. R. Bajaj, W. J. Mills, M. R. Lebo, B. Z. Hyatt and M. G. Burke, "Irradiation-Assisted Stress Corrosion Cracking of HTH Alloy X-750 and Alloy 625 (U)," presented at the Seventh International Symposium on Environmental Degradation of Materials in Nuclear Power Systems - Water Reactors, NACE, Vol. 2, 1995, p.p. 1093-1107

6. R. M. Boothby, J. Nucl. Matl. 186 (1992) 209.

7. E. Wimmer, H. Krakauer, M. Weinert and A. J. Freeman, Phys. Rev. B 24, 864 (1981)

8. J. P. Perdew, K. Burke and M. Ernzenhof, Phys. Rev. Lett. 77, 3865 (1996)

9. J. R. Rice and J. S. Wang, Mater. Sci. Eng. A107, 23 (1989). 
Table I. Strengthening Energies of Selected Impurities at a Nickel $\Sigma 5$ (210) Grain Boundary

Impurity Site Magnetic Case

$F$
$F$
$F$
$F$
$F$
$F$
$P$
$F$

S
Strengthening Energy (eV)

0.27

$-0.49$

0.70

1.25

2.50

7.26

7.21

1.20

\section{Effect}

Embrittling ${ }^{2}$

Strengthening ${ }^{2}$

Embrittling $^{2}$

Embrittling

Embrittling

Embrittling

Embrittling

Embrittling

Note 1: Data previously reported in Reference 4.

Note 2: Effect confirmed experimentally in Ni alloys

Site: I = extra atom in the GB; $S=$ substitutional impurity on the GB.

Magnetic Case: $F=$ Ferromagnetic; $P=$ Paramagnetic.

Figures

Fig. 1. Computational cells for FLAPW calculations. (a) Impurity at a Ni $\Sigma 5(210)$ grain boundary. (b) Impurities at the corresponding free surfaces after boundary cleavage. The structures are periodic in the horizontal direction and out of the page. 


\section{Vacuum}

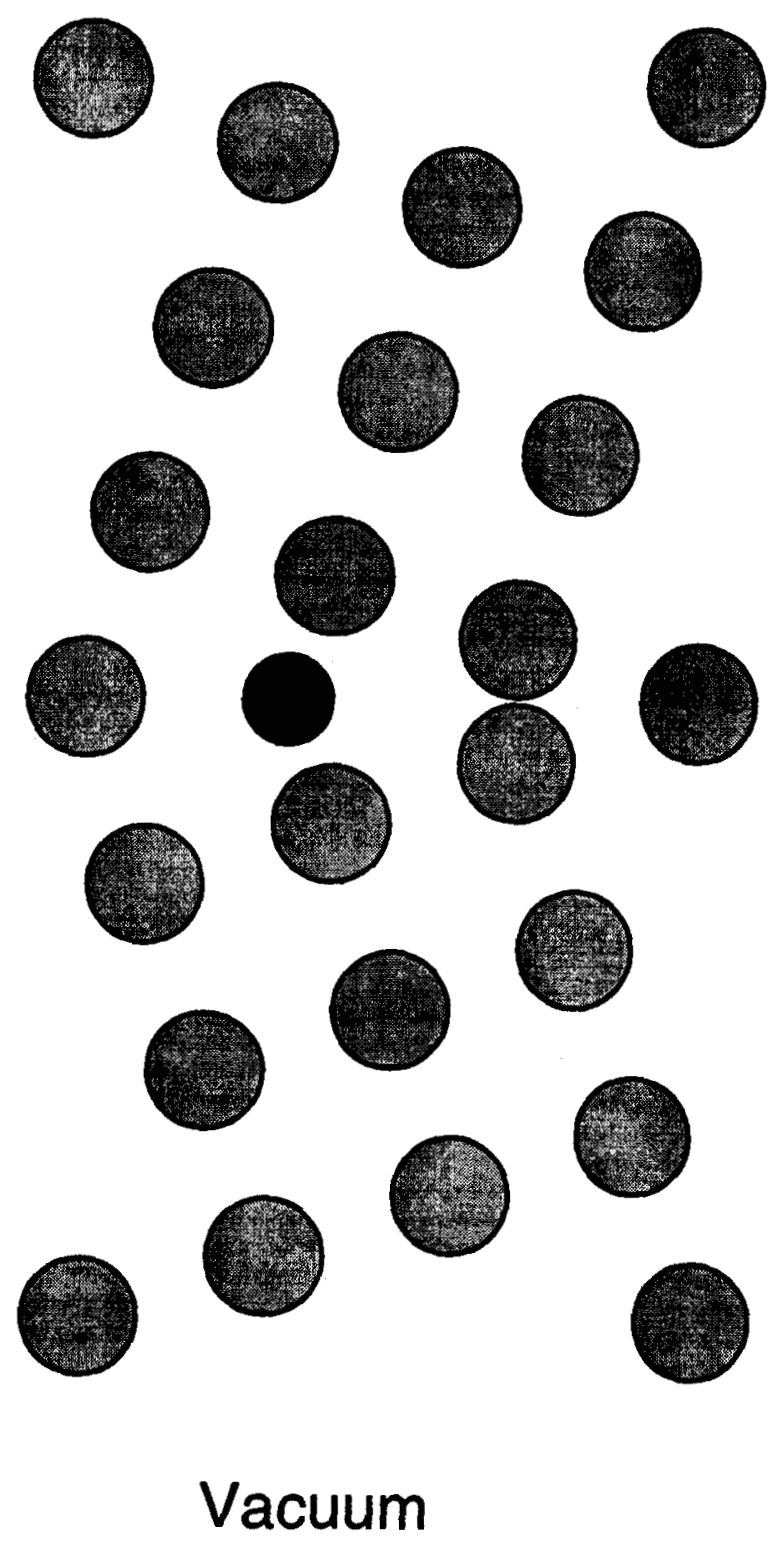

Fig.1 (GB) 


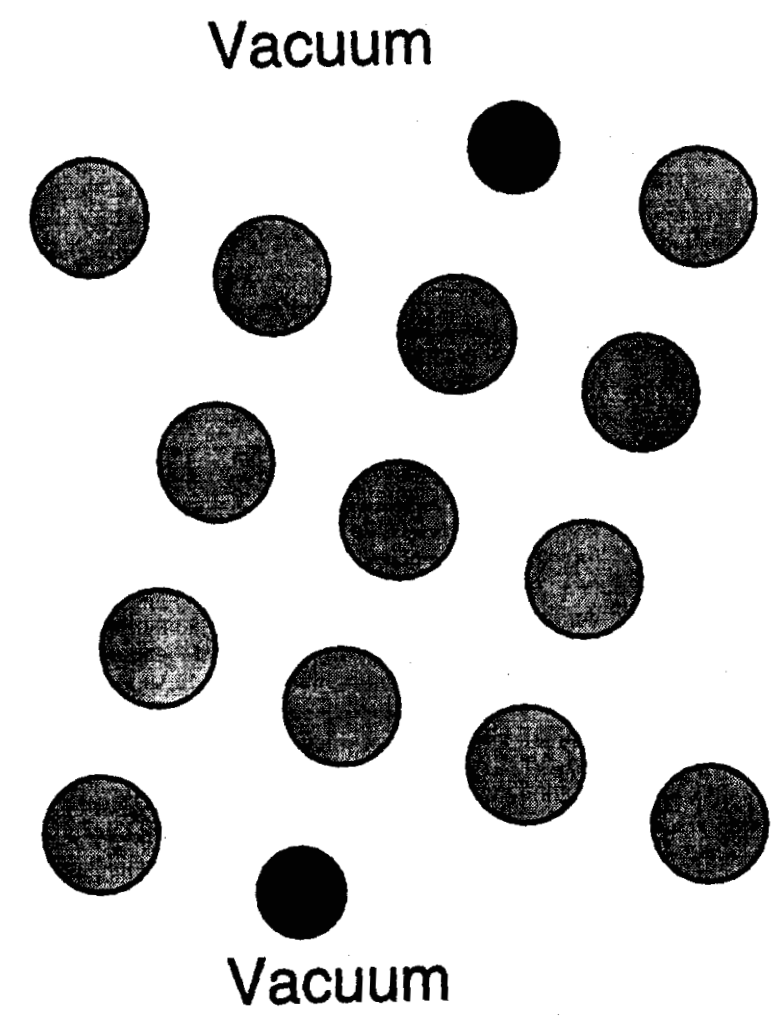

Fig.1 (FS) 